\section{Effects and adverse events after CXL for keratoconus are independent of age: a 1-year follow-up study}

PB Baenninger ${ }^{1}$, LM Bachmann ${ }^{2,3}$, L Wienecke ${ }^{1}$, C Kaufmann ${ }^{1}$ and MA Thiel ${ }^{1}$

\begin{abstract}
Purpose To compare the effect, failure rate and the risks of corneal cross-linking (CXL) in keratoconus patients aged $\geq 35$ years to patients $<35$ years.

Methods In 141 eyes of 116 keratoconus patients we compared the changes in best phoropter-corrected visual acuity (BCVA) and maximum keratometry values $\left(K_{\max }\right)$ before and 12 months after CLX in patients aged $\geq 35$ years ( $n=34,38$ eyes) to the cohort of patients below 35 years of age.
\end{abstract}

Results Overall, CXL significantly improved BCVA from 0.487 logMAR $(95 \%$ confidence interval (CI) 0.426-0.548) by - 0.197 logMAR (95\% CI -0.243 to $-0.150 ; P<0.001$ ) and reduced $K_{\max }$ from 48.96 diopter (Dpt) by $-1.33 \mathrm{Dpt}(95 \% \mathrm{CI}-1.85$ to $-\mathbf{0 . 8 1}$ : $P<0.001)$. Age $\geq 35$ years had no effect on the changes of BCVA $(-0.02(95 \%$ CI -0.13 to 0.09$) ; P=0.757)$ or $K_{\max }(0.58(95 \% \mathrm{CI}-0.51$ to 1.68$) ; P=0.294$ ) as compared with younger patients. In 54 patients (55 eyes, 38.5\%) aged $<35$ years and in 18 patients (18 eyes, $47.4 \%$ ) aged $\geq 35$ years, BCVA increased by $\geq 2$ Snellen lines. Failure (increase in $K_{\max }$ $\geq 1$ Dpt) was observed in 17 eyes (16.5\%) of patients aged $<35$ years and in 3 eyes (7.9\%) of patients aged $\geq 35$ years during the 12-month follow-up period. Adverse outcomes (loss of $\geq 2$ Snellen lines) occurred in 4 (3.9\%) eyes of patients aged $<35$ years and $1(2.6 \%)$ eye of a patient aged $\geq 35$ years.

Conclusion Effects and adverse events of CXL treatment do not seem to differ between subjects younger or older than 35 years. Eye (2014) 28, 691-695; doi:10.1038/eye.2014.56; published online 14 March 2014

\section{Introduction}

Keratoconus (KC) is a corneal ectasia that typically progresses in young adults, but the rate of progression slows with increasing age. ${ }^{1}$ Corneal cross-linking (CXL) using riboflavin and ultraviolet-A light has been shown to effectively increase corneal stiffness and even to reduce ectasia. ${ }^{2-4}$ These findings originate from studies done mainly in young adults and led to the widespread use of CXL to stabilize KC in this group of patients, who have a $20 \%$ risk of progressing to a stage that requires therapeutic keratoplasty.

Thus far, little attention has been paid to the effect of CXL in KC patients older than 35 years of age. During ageing, a natural cross-linking pathway occurs, which involves a nonenzymatic reaction. ${ }^{5}$ The physiological cross-linking of the cornea may explain the slowdown of KC progression in older patients. Despite the reduced rate of progression of $\mathrm{KC}$ with increasing age, $\mathrm{KC}$ can progress in older patients, resulting in reduced visual acuity and quality of life. ${ }^{1,6-8}$ Whether iatrogenic CXL in older adults with naturally cross-linked corneas has the same positive effect as in younger adults is unknown. A recent study on the safety of CXL found that age $\geq 35$ years was a major risk factor for adverse events after CXL. ${ }^{9}$ Hence, it is arguable whether CXL should be offered to KC patients aged $\geq 35$ years.

The aim of this study was to investigate the effect of CXL in KC patients aged $\geq 35$ years. In particular, we analyzed the effect of CXL on visual acuity, keratometric conus regression, and adverse events in patients aged $\geq 35$ years as compared with younger ones over a 12-month follow-up period.
'Department of Ophthalmology, Cantonal Hospital of Lucerne, Zug, Switzerland

${ }^{2}$ Medignition Inc. Healthcare Innovations, Zug, Switzerland

${ }^{3}$ Horten Center, University of Zurich, Zurich, Switzerland

Correspondence: PB Baenninger, Department of Ophthalmology, Cantonal Hospital of Lucerne, CH-6000 Lucerne-16, Switzerland Tel: +41 41205 3309; Fax: +41412053406. E-mail: philipp.baenninger@ luks.ch

Received: 29 April 2013 Accepted in revised form: 3 February 2014 Published online: 14 March 2014

Presented at the 114th Annual Meeting of American Academy of Ophthalmology (AAO), Chicago, IL, USA, 16-19 October 2010 


\section{Materials and methods}

\section{Study design}

This retrospective, single-centre study enrolled patients with mild-to-moderate $\mathrm{KC}\left(K_{\max }<65.00\right.$ diopter $)$ and a corneal thickness of $\geq 400 \mu \mathrm{m}$ at the time of surgery. Patients who did not fulfill these two criteria were excluded from this analysis. Indication for treatment was based on progression of KC. Progression was verified by repeated corneal topography over at least 6 months, and defined as an increase in the central maximum keratometry value $\left(K_{\max }\right)$ of $\geq 1.0$ diopter $(D p t)$. Progression was also accepted if patients needed a new contact lens fitting because of a steepening of the cone more than once in the last 2 years, in cases of diurnal fluctuation of vision, or if patients developed contact lens intolerance (three patients). Eligible patients were aged between 16 and 70 years, and were not pregnant or breastfeeding at the time of the treatment. Exclusion criteria were ocular pathology other than keratoconus and previous refractive or other corneal surgery.

\section{Examination}

Best phoropter-corrected visual acuity (BCVA) was assessed at the initial consultation and at follow-up visits at 1,3, and 12 months postoperatively using Snellen charts. $K_{\max }$ was also measured at the initial consultation and the 1-, 3-, and 12-moth postoperative follow-ups by corneal topography (Precisio iVIS Technologies, Taranto, Italy).

CXL failure was defined as the percentage of eyes with an increase in $K_{\max }$ of $>1.0$ Dpt 12 months after the intervention compared with the preoperative values.

The adverse event rate was defined as the percentage of eyes with a loss of $\geq 2$ Snellen lines of BCVA at the 12-month follow-up compared with preoperative values.

\section{Surgical technique}

After topical anesthesia (proxymetacaine hydrochloride $5 \mathrm{mg} / \mathrm{ml}$, Alcaine, Alcon Pharmaceuticals Ltd, Huenenberg, Switzerland) and insertion of a lid speculum, the epithelium was removed after a 10-s application of $94 \%$ ethanol (Laboratorium Dr Bichsel AG, Interlaken, Switzerland) in a 9.0-mm diameter area. The limbus was masked with instrument wipes (Visiwipe, Beaver-Visitec International, Waltham, MA, USA) to protect the stem cells from irradiation. Then $0.1 \%$ riboflavin, 20\% dextran drops (Medio-cross, Peschke $\mathrm{GmbH}$, Waldshut, Germany) were instilled every $3 \mathrm{~min}$ for $30 \mathrm{~min}$. Stromal thickness during CXL was monitored with ultrasonic pachymetry (Corneo-Gage, Sonogage, Cleveland, OH, USA). In the event that pachymetry readings dropped below $400 \mu \mathrm{m}$, a hypotonic riboflavin solution (Vitamin B2 Streuli, Streuli Pharma, Uznach, Switzerland) was used to increase stromal thickness. ${ }^{10}$ Penetration of riboflavin into the aqueous humor was verified by slit lamp examination (blue light) before irradiating the eye for $30 \mathrm{~min}$ with ultraviolet-A light $(370 \mathrm{~nm})$ with an irradiance of $3 \mathrm{~mW} / \mathrm{cm}^{2}$ (UV-X System; Peschke Meditrade $\mathrm{GmbH}$, Huenenberg, Switzerland). During irradiation, $0.1 \%$ riboflavin drops were instilled every $5 \mathrm{~min}$ and further anesthetic drops if needed by the patient. After the procedure, a bandage contact lens (Air Optix Night\&Day, Ciba Vision AG, Embrach, Switzerland) was applied until re-epithelialisation was complete on postoperative day 4. Topical antibiotic (ofloxacin $3 \mathrm{mg} / \mathrm{ml}$, Floxal UD, Bausch\&Lomb, Zug, Switzerland) 4 times daily was given for 5 days, the use of lubricating eye drops (Lacrycon SDU, Thea Pharma SA, Schaffhausen, Switzerland) was at the patient's discretion. After epithelial healing, patients used topical fluorometholone (FMLLiquifilm, Allergan AG, Freienbach, Switzerland) 5 times daily for 4 weeks.

\section{Statistical analysis}

Interval scaled variates were summarized with means and SD or medians and interquartile ranges, where appropriate. Dichotomous variates were described as ratios and percentages. For statistical reasons, we expressed visual acuity with the logMAR (logarithm of the minimum angle of resolution $=$ minus natural logarithm of BCVA). For both outcome parameters $\log M A R$ and $K_{\max }$, we first calculated the difference $(\log M A R$ after CLX - logMAR before CLX) and ( $K_{\max }$ before CLX $-K_{\max }$ after CLX). To explore the strength of the association between $\log$ MAR or $K_{\max }$ changes and patients' age $\geq 35$ years, we fitted two multivariate mixed linear models using subject as a random factor. These models adjusted for the fact that measurements were independent between subjects but dependent within subjects if a subject provided data on both eyes. This approach thus allowed analyzing data of all available eyes validly. To each model we entered an indicator variate for age $\geq 35$ years. To adjust for potential confounders identified in a previous study, ${ }^{9}$ we also added indicator variates for preoperative BCVA better than 20/25 and preoperative maximum $\mathrm{K}$ readings $>58.00 \mathrm{Dpt}$. All analyses were performed using the Stata 11.1 statistics software package (StataCorp LP, College Station, TX, USA).

\section{Results}

\section{Patients' characteristics}

During the study period between January 2007 and January 2010, 166 eyes of 141 patients received CXL 
treatment for $\mathrm{KC}$ at our institution. During the 12-month follow-up period, 25 eyes of 25 patients were lost to follow-up. Hence, the final analysis included 141 cross-linked eyes of 116 KC patients. Detailed patient characteristics are given in Table 1.

\section{Overall effects of CXL}

For the overall population, CXL improved BCVA significantly from $0.487 \log$ MAR (95\% CI $0.426-0.548)$ by -0.197 logMAR $(95 \% \mathrm{CI}-0.243$ to $-0.150 ; P<0.001)$. The maximum $\mathrm{K}$ values regressed by -1.33 (95\% CI -1.85 to $-0.81: P<0.001)$ during the 12 -month follow-up period.

\section{Effect of age on CXL}

In $\mathrm{KC}$ patients undergoing CXL, age $\geq 35$ years had no effect on the changes of BCVA $(-0.02(95 \% \mathrm{CI}-0.13$ to $0.09) ; P=0.757)$ or $K_{\max }(0.58$ (95\% CI -0.51 to 1.68$)$; $P=0.294)$ as compared with patients $<35$ years of age even when correcting for potential confounders such as BCVA better than 20/25 and preoperative maximum $\mathrm{K}$ readings $>58.00 \mathrm{Dpt}$. In 54 patients (55 eyes, 38.5\%) aged $<35$ years, and in 18 patients (18 eyes, 47.4\%) aged $\geq 35$ years, BCVA increased by $\geq 2$ Snellen lines. Also, for $K_{\max }$ we found 12 months after CXL a nonsignificant interaction of $-0.515(95 \% \mathrm{CI}-1.610$ to $0.580: P=0.354)$ indicating a slightly larger reduction of $K_{\max }$ in patients aged $\geq 35$ years as compared with patients $<35$ years (Figure 1).

\section{Treatment failures and rate of adverse events}

Treatment failures (defined as increase in $K_{\max } \geq 1 \mathrm{Dpt} 12$ months after CXL) were observed in 17 eyes (16.5\%) in patients aged $<35$ years, and in 3 eyes $(7.9 \%)$ of patients aged $\geq 35$ years (Fisher's exact test $P=0.278$ ).
Adverse events of CXL (loss of $\geq 2$ Snellen lines) were seen in 4 eyes $(3.9 \%)$ of patients aged $<35$ years and 1 eye $(2.6 \%)$ of a patient aged $\geq 35$ years.

The overall findings did not change when excluding data of the second eye in patients providing data on both eyes.

\section{Conclusion}

In this 12-month CXL follow-up study, improvement of BCVA and $K_{\max }$ and the number of adverse events did not seem to differ between subjects younger or older than 35 years.

Since the introduction of CXL over 10 years ago, several studies ${ }^{4,11}$ proved the benefit of stopping $\mathrm{KC}$ progression with CXL. The effect of CXL found in our patients with regard to improved visual acuity and reduced keratometry is very similar to all other studies investigating the effect of CXL with epithelial removal in KC patients. $^{2-4,11,12}$

An important objective of this study was to assess the risk of adverse events after CXL. Despite the overall enthusiasm for offering CXL to KC patients, adverse events have been incompletely assessed in the literature. ${ }^{13,14}$ Koller et $a l^{9}$ reported an overall adverse event rate of $2.9 \%$ in 105 eyes, and identified age $\geq 35$ years as the most important independent risk factor for adverse events after CXL. However, this findings needs to be interpreted cautiously, because, given the figures provided in the paper, the estimated number of eyes in patients aged $>35$ years enrolled in the Koller study was only 15 with 2 failures, corresponding to a mean probability of 0.13 and a binomial exact $95 \%$ CI of $0.02-0.40$.

Our study in 141 eyes showed an adverse event rate of $3.9 \%$ in 82 patients (103 eyes) aged $<35$ years $v$ s $2.6 \%$ in 34 patients ( 38 eyes) aged $\geq 35$ years. All adverse events were due to stromal haze formation, which induced a

Table 1 Group characteristics before CXL

\begin{tabular}{|c|c|c|c|c|}
\hline Parameter & All $(\mathrm{n}=116,141$ eyes $)$ & $\begin{array}{l}\text { Group }<35 \text { years of age } \\
\quad(\mathrm{n}=82 ; 103 \text { eyes })\end{array}$ & $\begin{array}{l}\text { Group } \geq 35 \text { years of age } \\
\quad(\mathrm{n}=34 ; 38 \text { eyes })\end{array}$ & P-values \\
\hline Mean age (SD) & $29.8(10.3)$ & $24.8(5.1)$ & $43.4(8.6)$ & - \\
\hline Male patients $(\%)$ & $86(74.5 \%)$ & $65(79.6 \%)$ & $21(60.5 \%)$ & 0.021 \\
\hline Median (IQR) BCVA & $0.40(0.22-0.70)$ & $0.30(0.22-0.70)$ & $0.40(0.30-0.70)$ & - \\
\hline Median (IQR) $K_{\max }(\mathrm{Dpt})$ & $48.2(44.8-52.1)$ & $47.8(44.8-51.8)$ & $49.5(44.5-52.8)$ & - \\
\hline Mean (SD) pachymetry $(\mu \mathrm{m})$ & $445.8(47.3)$ & $447.8(44.9)$ & $440.5(53.6)$ & 0.422 \\
\hline Patients with $K_{\max }>58 \mathrm{Dpt}$ & $7 / 116$ & $5 / 82$ & $2 / 34$ & 0.965 \\
\hline \multirow[t]{3}{*}{ Preferred everyday optical correction } & Contact lens $(n=26)$ & 19 & 7 & $<0.001$ \\
\hline & Spectacles $(n=91)$ & 66 & 25 & 0.036 \\
\hline & None $(n=24)$ & 18 & 6 & 0.056 \\
\hline $\begin{array}{l}\text { Additional hypo-osmotic riboflavin in eyes with } \\
\text { central corneal pachymetry of }<400 \mu \mathrm{m}\end{array}$ & 21 eyes & $14 / 103(13.6 \%)$ & $7 / 38(18.4 \%)$ & 0.475 \\
\hline
\end{tabular}

Abbreviations: $\mathrm{CXL}$, corneal cross-linking; $\mathrm{IQR}=$ interquartile range. 

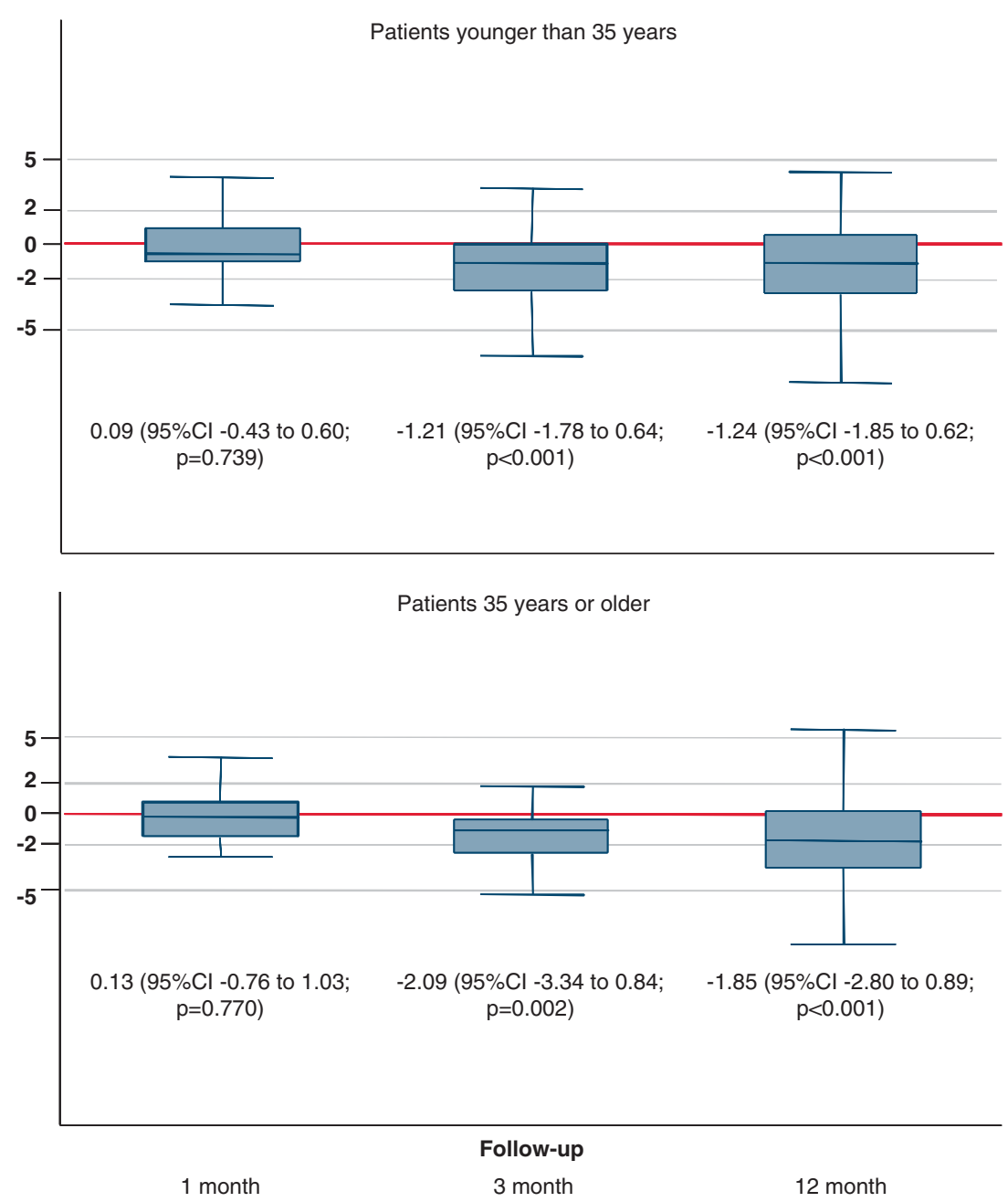

Figure 1 Change in maximum central keratometry $\left(K_{\max }\right)$ compared with baseline at the 1-, 3-, and 12-month follow-up visits in patients aged $<35$ years and $\geq 35$ years.

loss of BCVA, except for one case in a young patient where a contact lens-induced epithelial problem was the reason for vision deterioration at the 12-month followup. Taking into account that stromal haze formation is a common finding after CXL ${ }^{15}$ and, because none of the patients experienced a severe complication, we still regard $\mathrm{CXL}$ as a relatively safe procedure in all age groups.

A limitation of our study is that the failure rate (7.9-16.5\%), defined as increase of $K_{\max } \geq 1$ Dpt after $\mathrm{CXL}$, was somewhat higher than the failure rate reported in the literature (0-7.6\%).9,11,12,16 Arguably, the rate depends on the inclusion criteria, and further investigations are needed to define factors predicting the risk of failure. Including patients with high $K_{\max }$ values could be one reason for the higher rate of failures. In our sample, however, this might not be relevant, because only seven patients with $K_{\max }>58 \mathrm{Dpt}$ were included and only one of them experienced a failure. Regarding the rate of adverse events, we did not objectively assess the extent of haze, which was the main cause for the persistent loss of vision after CXL. However, all clinical patient assessments were performed by the same experienced clinician, thereby limiting the extent of variability in judgment.

A potential problem of this retrospective analysis is the $25(15.1 \%)$ patients lost to follow-up not attending the 12-month follow-up visit used to analyze the outcome data. We tried to contact the 25 lost patients by phone. We were able to reach 19 patients (14 aged $<35$ years and 5 aged $\geq 35$ years). All 19 patients stated that they had missed their 1-year follow-up visit because they were satisfied with the outcome after CXL. Eighteen patients said that their vision had subjectively stayed unchanged or improved as compared with the time before CXL. One patient (aged 16 years) reported a slightly reduced vision, which did not bother him enough to attend the follow-up visit. We were unable to contact 6 patients ( 4 aged $<35$ years and 2 aged $>35$ years). Overall, 
patients' feedback makes us confident that the substantial loss to follow-up has not biased our results substantially.

Because of the low number of adverse events, further prospective studies or a meta-analysis with larger patient numbers will be required to analyze the safety and complication rate of CXL. Using the approach of Blackwelder, ${ }^{17}$ and assuming that a difference in adverse effects between the two age groups of $5 \%$ or less would mean equivalence, the required sample size would be 283 patients per group with a power of $80 \%$ and an alpha level of $5 \%$.

By assessing stromal haze formation after CXL and comparing stromal haze with BCVA, the complication rate might be reduced even more. Additional data may also help identify possible risk factors for failure of the intervention.

In conclusion, CXL improves visual acuity and reduces maximal keratometry values in $\mathrm{KC}$ patients aged $\geq 35$ years as effectively as in younger patients. In addition, age did not seem to be a risk factor for adverse events, neither were preoperative BCVA better than 20/25 Snellen and a preoperative maximum $\mathrm{K}$ reading >58.00 Dpt. Therefore, CXL should be considered a valuable treatment option in patients aged $\geq 35$ years, especially if they experience increasing CL intolerability or reduced visual acuity.

\section{Summary}

\section{What was known before}

- Keratoconus is a corneal ectasia that typically progresses in young adults and is typically treated with crossliniking. Thus far, little attention has been paid to visual acuity, keratometric conus regression, and adverse events of cross-liniking in keratoconus patients $>35$ years of age.

\section{What this study adds}

- Improvement of visual acuity keratometric conus regression and the number of adverse events do not seem to differ between subjects younger or older than 35 years. Cross-liniking should be considered a valuable treatment option in patients aged $\geq 35$ years, especially if they experience increasing contact lens intolerability or reduced visual acuity.

\section{Conflict of interest}

The authors declare no conflict of interest.

\section{References}

1 Wagner H, Barr JT, Zadnik K. Collaborative Longitudinal Evaluation of Keratoconus (CLEK) Study: methods and findings to date. Cont Lens Anterior Eye 2007; 30(4): 223-232.
2 Coskunseven E, Jankov 2nd MR, Hafezi F. Contralateral eye study of corneal collagen cross-linking with riboflavin and UVA irradiation in patients with keratoconus. J Refract Surg 2009; 25(4): 371-376.

3 Hersh PS, Greenstein SA, Fry KL. Corneal collagen crosslinking for keratoconus and corneal ectasia: one-year results. J Cataract Refract Surg 37(1): 149-160.

4 Wittig-Silva C, Whiting M, Lamoureux E, Lindsay RG, Sullivan LJ, Snibson GR. A randomized controlled trial of corneal collagen cross-linking in progressive keratoconus: preliminary results. J Refract Surg 2008; 24(7): S720-S725.

5 Malik NS, Moss SJ, Ahmed N, Furth AJ, Wall RS, Meek KM. Ageing of the human corneal stroma: structural and biochemical changes. Biochim Biophys Acta 1992; 1138(3): 222-228.

6 Davis LJ, Schechtman KB, Wilson BS, Rosenstiel CE, Riley $\mathrm{CH}$, Libassi DP et al. Longitudinal changes in visual acuity in keratoconus. Invest Ophthalmol Vis Sci 2006; 47(2): 489-500.

7 Kymes SM, Walline JJ, Zadnik K, Sterling J, Gordon MO. Changes in the quality-of-life of people with keratoconus. Am J Ophthalmol 2008; 145(4): 611-617.

8 McMahon TT, Szczotka-Flynn L, Barr JT, Anderson RJ, Slaughter ME, Lass JH et al. A new method for grading the severity of keratoconus: the Keratoconus Severity Score (KSS). Cornea 2006; 25(7): 794-800.

9 Koller T, Mrochen M, Seiler T. Complication and failure rates after corneal crosslinking. J Cataract Refract Surg 2009; 35(8): 1358-1362.

10 Hafezi F, Mrochen M, Iseli HP, Seiler T. Collagen crosslinking with ultraviolet-A and hypoosmolar riboflavin solution in thin corneas. J Cataract Refract Surg 2009; 35(4): 621-624.

11 Wollensak G, Spoerl E, Seiler T. Riboflavin/ultraviolet-ainduced collagen crosslinking for the treatment of keratoconus. Am J Ophthalmol 2003; 135(5): 620-627.

12 Raiskup-Wolf F, Hoyer A, Spoerl E, Pillunat LE. Collagen crosslinking with riboflavin and ultraviolet-A light in keratoconus: long-term results. J Cataract Refract Surg 2008; 34(5): 796-801.

13 Herrmann CI, Hammer T, Duncker GI. [Hazeformation (corneal scarring) after cross-linking therapy in keratoconus]. Ophthalmologe 2008; 105(5): 485-487.

14 Mazzotta C, Balestrazzi A, Baiocchi S, Traversi C, Caporossi A. Stromal haze after combined riboflavin-UVA corneal collagen cross-linking in keratoconus: in vivo confocal microscopic evaluation. Clin Experiment Ophthalmol 2007; 35(6): 580-582.

15 Greenstein SA, Fry KL, Bhatt J, Hersh PS. Natural history of corneal haze after collagen crosslinking for keratoconus and corneal ectasia: Scheimpflug and biomicroscopic analysis. J Cataract Refract Surg 36(12): 2105-2114.

16 Caporossi A, Baiocchi S, Mazzotta C, Traversi C, Caporossi T. Parasurgical therapy for keratoconus by riboflavin-ultraviolet type A rays induced cross-linking of corneal collagen: preliminary refractive results in an Italian study. J Cataract Refract Surg 2006; 32(5): 837-845.

17 Blackwelder WC. 'Proving the null hypothesis' in clinical trials. Control Clin Trials 1982; 3(4): 345-353. 